\title{
Transmission Schedule Optimization for Half-Duplex Multiple-Relay Networks
}

\author{
Wei Wang \\ Department of Electrical and \\ Computer Engineering \\ National University of Singapore \\ Email: wang.wei@nus.edu.sg
}

\author{
Lawrence Ong \\ School of Electrical Engineering \\ and Computer Science \\ The University of Newcastle \\ Email: lawrence.ong@cantab.net
}

\author{
Mehul Motani \\ Department of Electrical and \\ Computer Engineering \\ National University of Singapore \\ Email: motani@nus.edu.sg
}

\begin{abstract}
Half duplex devices are widely used in today's wireless networks. These devices can only send or receive, but not do both at the same time. In this paper, we use cooperative decode-forward relay strategies to increase the throughput of half-duplex wireless networks. Due to the half duplex constraint, relays need to carefully choose their transmission states in order to maximize the throughput. We show that the transmission schedule optimization can be formulated as a linear programming problem. Although the number of possible states grows exponentially as the number of relays increases, only a small subset of these states needs to be used in the optimal transmission schedule. This observation allows us to use heuristic algorithms to solve for near-optimal schedule in large networks. Our numerical results show that the decode-forward strategy can provide nearly 3 times more throughput than the traditional multi-hop relaying strategy in half duplex wireless networks.
\end{abstract}

Index Terms-Multiple relay channel, half-duplex, Optimization.

\section{INTRODUCTION}

New applications in wireless networks, such as video conferences and mobile TV, are demanding higher data rates over wireless links. Most researchers in wireless networking assume that packets are transmitted through point-to-point links in a multi-hop fashion, where concurrent transmitting links will inevitably interfere with each other [1], [2], [3]. Under the point-to-point wireless link model, interference between neighboring nodes leads to fundamental limitations on the achievable throughput of wireless ad hoc networks [1].

Studies in information theory show that nodes in wireless networks can cooperatively send and receive messages instead of simply interfering with each other. In the relay channel studied in information theory [4], relay nodes and the source node can cooperate with each other by transmitting to the destination at the same time. The destination then combines signals received from multiple nodes to decode the original message. Recent research shows that hierarchical cooperation can achieve optimal capacity scaling in wireless networks [5]. Therefore, such cooperative communication could be a promising way to increase the throughput of wireless networks.

On the other hand, cooperation also means higher costs. In information theory literature, the relay channel [6], [7], [8] is studied assuming full duplex nodes, i.e., nodes can listen and transmit at the same time on the same frequency band. However, most of the radio frequency transceivers used today either operate in half-duplex mode or in full duplex mode with the transmitter and the receiver operating on separate frequency bands (which can be modeled as orthogonal channels [9]). Therefore, it is important to consider more practical half-duplex relays which can either listen or transmit, but not do both at the same time.

In this paper, we focus on half-duplex relays using the decode-forward (DF) strategy. Instead of studying the singlerelay channel [10], [11], [12], [13], we consider the multiplerelay channel (MRC), which has one source/destination pair and multiple relays. In this case, a relay receives and combines signals from upstream nodes to decode the source message. After fully decoding the source message, the relay then sends out signals to help downstream nodes decode the message. As the relays cannot transmit and listen at the same time, they should balance the time fractions spent in listening and transmitting. Relays should spend enough time in the listening state in order to decode the message. On the other hand, they also need to transmit as much as possible to help downstream nodes. Moreover, the receiving rate of a node depends on the transmitting/listening states of all upstream nodes on the route. Therefore, the optimal transmission state for one relay depends on the transmission states of all other relays on the route.

The transmission state scheduling problem aims to find an optimal combination of states so that the throughput is maximized. When there are $M$ relays on the route, the number of possible combinations of transmission states is $2^{M}$. The exponential growth in the number of states makes the optimization problem formidable at first glance. However, we show that the optimal schedule only needs to use at most $M+1$ states to achieve the maximum rate for the DF strategy. This observation greatly simplifies the scheduling problem. We propose a heuristic algorithm which can find schedules that achieve an average rate within $99.97 \%$ of the optimal rate.

The achievable rates of the half-duplex DF scheme are also compared with rates of the cut-set upper bound and the multi-hop scheme via intensive numerical experiments. The numerical results show that the cooperative DF relay scheme can provide 2.8 times higher rate than conventional multi-hop scheme in a string topology. 
The rest of this paper is organized as follows. Section II introduces the channel model for the phase fading Gaussian relay channel and gives the achievable rate of the half-duplex DF scheme. We then formulate the transmission state optimization problem as a linear programming problem and solve it via the cutting plane method in section III. The performance of the DF scheme is compared with the cut-set bound and the multi-hop scheme in section IV. Finally, section V concludes the whole paper.

\section{Half-Duplex Multiple-Relay Networks}

\section{A. Multiple-Relay Channel (MRC)}

We consider the multiple-relay channel (MRC) with one source/destination pair and multiple relays in between, which has been studied by Gupta and Kumar [14], and Xie and Kumar [6]. It is an extension of the single-relay channel (SRC) [15] to the case where there are multiple relays helping the source and destination.

An $M$-relay $\mathrm{MRC}$ has $M+2$ nodes $\{0,1,2, \ldots, M, M+$ $1\} \triangleq \mathcal{M}$. The message $W$ is generated by the source (node 0 ) and is to be sent to the destination (node $M+1$ ) with the helps of $M$ relays (nodes 1 to $M$ ). We denote the set of all relay nodes as $\mathcal{R}$.

In the half-duplex MRC, nodes can only transmit or listen, but not do both, at any point in time. We assume that the source node is always transmitting and the destination node is always listening. To capture the half-duplex scenarios among the relays, we define the transmission state vector as follows.

Definition 1: For the $M$-relay MRC where the source always transmits and the destination always listens, the transmission state vector can be expressed as $\mathbf{s}=\left(s_{1} s_{2} \ldots s_{M}\right)$, where $s_{i}=1$ when node $i$ transmits and $s_{i}=0$ when node $i$ listens in state $\mathbf{s}$.

The $i$-th position in $\mathbf{s}$ indicates whether relay $i$ is listening or transmitting in state $\mathbf{s}$. Note that the transmission state vectors are the binary representation for integers from 0 to $2^{M}-1$. In the remainder of this paper, we will directly use integers in $\left[0,2^{M}-1\right]$ to represent the state $\mathbf{s}$. We assume that the transmission state for all channel uses are known to all nodes a priori.

Definition 2: For a transmission state $\mathbf{s}$, we define $\mathcal{L}(\mathbf{s})$ as the set of nodes that are listening and $\mathcal{T}(\mathbf{s})$ as the set of nodes that are transmitting. This means $\mathcal{L}(\mathbf{s}) \triangleq\left\{i \in \mathcal{R}: s_{i}=\right.$ $0\} \cup\{M+1\}$ and $\mathcal{T}(\mathbf{s}) \triangleq\left\{i \in \mathcal{R}: s_{i}=1\right\} \cup\{0\}$, where $\mathcal{R}=\{1,2, \ldots, M\}$ is the set of all relays.

\section{B. Half-Duplex Phase Fading Gaussian MRC}

Now we define the half-duplex phase fading $M$-relay Gaussian MRC. In this paper, we only consider memoryless and time invariant channels [7]. We use $x_{i}$ to denote an input from node $i$ into the channel and $y_{i}$ denotes an output from the channel to node $i$. For nodes that are transmitting, i.e., $i \in \mathcal{T}(\mathbf{s})$, we set $y_{i}=0$. For nodes that are listening, i.e., $i \in \mathcal{L}(\mathbf{s})$, we set $x_{i}=0$. During state $\mathbf{s}$, the received signal at node $k$ is given by

$$
Y_{k}=\left\{\begin{array}{ll}
\sum_{i \in \mathcal{T}(\mathbf{s}) \backslash\{k\} \sqrt{\lambda_{i, k}} e^{j \theta(i, k)} X_{i}+Z_{k}} \quad k \in \mathcal{L}(\mathbf{s}) \\
0 & k \in \mathcal{T}(\mathbf{s})
\end{array},\right.
$$

where $X_{i}$, the input to the channel from node $i$, is a zero-mean complex random variable. $Z_{k}$, the receiver noise at node $k$, is an i.i.d., zero-mean, complex, Gaussian random variable with variance $E\left[Z_{k} Z_{k}^{\dagger}\right]=N_{k} . \lambda_{i, k}$, capturing the path loss from node $i$ to node $k$, is defined as

$$
\lambda_{i, k}=\left\{\begin{array}{ll}
\kappa d_{i, k}^{-\eta} & d_{i, k} \geq 1 \\
\kappa & \text { otherwise }
\end{array},\right.
$$

where $d_{i, k} \geq 0$ is the distance between nodes $i$ and $k, \eta \geq 2$ is the path-loss attenuation exponent, and $\kappa$ is a positive constant. $e^{j \theta(i, k)}$ is the phase fading random variable, where $\theta(i, k)$ is uniformly distributed over $[0,2 \pi) . \theta(i, k)$ for all $i$ and $k$ are jointly independent of each other.

We assume that all nodes know $\kappa$ and $d_{i, k}$. We also assume that node $k$ only knows $\theta(i, k), \forall i$ and does not know any $\theta(i, l)$ for $l \neq k$. Hence, the transmitted signals of node $i$ cannot be chosen as a function of $\theta(i, k)$ for any $k$.

In this paper, we consider the following per-symbol power constraint on individual nodes. With the half duplex constraints, $x_{i}=0$ for node $i$ in the listening state, we have the power constraint as

$$
E\left[X_{i} X_{i}^{\dagger}\right] \leq\left\{\begin{array}{ll}
P_{i} & i \in \mathcal{T}(\mathbf{s}) \\
0 & i \in \mathcal{L}(\mathbf{s})
\end{array} .\right.
$$

\section{Achievable Rates in Phase Fading Gaussian MRC}

Define the route as $\mathcal{O}=\left\{o_{0}, o_{1}, \ldots, o_{|\mathcal{O}|-1}\right\}$, which is an ordered set of nodes from the source to the destination [16], [17]. We always set $o_{0}$ as node 0 (the source) and $o_{|\mathcal{O}|-1}$ as node $M+1$ (the destination). Given the route $\mathcal{O}$ and the transmission state $\mathbf{s}=j$, a node $o_{i}$ with $i \geq 1$ on the route can decode from the signals sent by all nodes in the upstream of the route which are transmitting in state $\mathbf{s}=j$. Therefore, in the phase fading Gaussian MRC, a node $o_{i}$ can receive at a rate of [18]:

$r_{o_{i}, j}= \begin{cases}\log \left(1+\frac{\sum_{k \in\left(o_{0}, o_{1}, \ldots, o_{i-1}\right) \cap \mathcal{T}(j)} \lambda_{k, o_{i}} P_{k}}{N_{o_{i}}}\right) & o_{i} \in \mathcal{L}(j) \\ 0 & o_{i} \in \mathcal{T}(j)\end{cases}$

when transmission state $\mathbf{s}=j$.

Define a transmission schedule $\mathcal{Q}=\left\{q_{0}, q_{1}, \ldots, q_{2^{M}-1}\right\}$, where $\sum_{j=0}^{2^{M}-1} q_{j}=1$. Consider a block coding scheme and define each $m$ uses of the channel as a block. In a schedule $\mathcal{Q}$, nodes will use a transmission state $\mathbf{s}=j$ for a time fraction of $q_{j}$ in each block.

A node $o_{i}$ will use all information it received during blocks $n-i+1$ to $n$ for decoding the $(n-i+1)$ th block sent by the source. For example, when the source is sending block 2, the first relay $o_{1}$ should be able to decode the information of block 2 . During the same block, the first relay should also send 
information about block 1 to downstream nodes. The second relay $o_{2}$, should have received information about block 1 from both the source $o_{0}$ and the first relay $o_{1}$. So it can combine both information to decode block 1 . In this way, the message $W$ can be sent in a pipelined manner through multiple blocks along the route [18].

In [18], we proved that there exists a coding scheme which can achieve rates up to

$$
R_{\mathrm{DF}}=\max _{\mathcal{O} \in \Pi(\mathcal{M})} \max _{\mathcal{Q}} \min _{o_{i} \in \mathcal{O} \backslash\{0\}} \sum_{0 \leq j \leq 2^{M}-1} q_{j} r_{o_{i}, j}
$$

in the half-duplex phase fading Gaussian MRC, where $\prod(\mathcal{M})$ is the set of all combinations of routes from the source to the destination. The achievable DF rate is the maximum rate over all possible routes and transmission schedule selections. The third minimization requires that the destination node and all relay nodes should be able to fully decode the message. The first maximization, i.e., finding an optimal relay route $\mathcal{O}^{*}$, has been studied in [17]. In this paper, we focus on finding the optimal transmission schedule which can achieve the maximum $\mathrm{DF}$ relay rate when the route $\mathcal{O}$ is given as $o_{i}=i, \forall i \in[0, M+1]$.

\section{OPTIMIZATION ON TRANSMISSION SCHEDULE}

In the DF strategy, every relay should be able to fully decode the message sent by the source. Thus, half-duplex relays must find the optimal transmission schedule which decides how long they should listen and in which transmission states they should listen. As the message is sent in a block coding scheme, it can be proven that the achievable rate only depends on the time fraction of transmission states used in the block, but not on the order of the states [18]. Therefore, the values for $q_{j}$ can fully describe an optimal transmission schedule in half-duplex MRC.

\section{A. Linear Programming Solution}

The optimization over transmission schedule can be formulated as a linear programming problem as follows:

$$
\begin{aligned}
& \text { Maximize } u \text {, } \\
& \text { s.t. } u-\sum_{j=0}^{2^{M}-1} q_{j} r_{i, j} \leq 0 \quad 1 \leq i \leq M+1 \\
& \sum_{j=0}^{2^{M}-1} q_{j}=1 \\
& q_{j} \geq 0 \text {. }
\end{aligned}
$$

The decision variables in this linear programming are $u$ and $q_{j}$ s. Constraints (6) require that all relays and the destination should have a receiving rate, summed over all transmission states, to be larger than $u$. Therefore, maximizing $u$ is equivalent to maximizing $\min _{1 \leq i \leq M+1}\left\{\sum_{j} q_{j} r_{i, j}\right\}$ [19]. In this way, we can guarantee that node $o_{i}$ can always decode block $n-i+1$ when the source is sending block $n$. Constraint (7) requires the sum of time fractions of all used states should be equal to one.
In an MRC with $M$ relays, the number of possible transmission states is $2^{M}$. Therefore, the number of variables in this linear programming increases exponentially with $M$ and it is difficult to directly solve this problem in large networks. However, we observe that there are only $M+2$ constraints in (6) and (7). Consequently, every basic feasible solution for the linear programming only has $M+2$ non-zero variables. As the variable $u$ in a non-trivial solution is always non-zero, there are at most $M+1$ non-zero time fractions $q_{j}$ in the optimal schedule. So, among all the $2^{M}$ possible states, only $M+1$ states will be used in the optimal schedule $\mathcal{Q}^{*}$. As we only need to pick up $M+1$ states among the $2^{M}$ states, the optimization process could be greatly simplified.

This observation also shows that the scheduling scheme may be practical in real networks. Since we only need to use a small number states in the schedule, the coordination and code design for multiple relays could also be simplified.

\section{B. Cutting Plane Method}

The cutting plane method can use iterative algorithms to solve the linear optimization problem proposed in last section [19]. Consider the dual of the linear programming problem in (6) - (8):

$$
\begin{array}{ll} 
& \text { Minimize } v, \\
\text { s.t. } & v-\sum_{i=1}^{M+1} w_{i} r_{i, j} \geq 0 \quad 0 \leq j \leq 2^{M}-1 \\
& \sum_{i=1}^{M+1} w_{i}=1 \\
& w_{i} \geq 0 .
\end{array}
$$

The decision variables in the dual problem are $v$ and $w_{i} \mathrm{~s}$. We see that the dual problem only has $M+2$ variables. However, the number of constraints in (9) is $2^{M}$.

In the relaxed dual problem, we only consider a small subset of the $2^{M}$ constraints in (9). It is easy to see that each constraint in (9) corresponds to one transmission state in the schedule. Thus, the relaxed dual problem only considers a subset $\mathcal{J} \subseteq\left\{0,1, \ldots, 2^{M}-1\right\}$ of all the possible states. Suppose the optimal solution of the relaxed dual problem is $\left[\begin{array}{ll}v^{*} & \mathbf{w}^{*}\end{array}\right]$. If the $v^{*}$ and $\mathbf{w}^{*}$ satisfy constraints (9) for all states, i.e., $v^{*} \geq \sum_{i} w_{i}^{*} r_{i, j}, \forall j \in\left[0,2^{M}-1\right]$, the solution of $\left[\begin{array}{ll}v^{*} & \mathbf{w}^{*}\end{array}\right]$ is then a feasible and optimal solution to the original dual problem. Otherwise, we can always find a state $k$ where constraint (9) is violated, i.e., $v^{*}<\sum_{i} w_{i}^{*} r_{i, k}$. In this case, we can include state $k$ into $\mathcal{J}$ and the optimal $v^{*}$ for the new relaxed dual problem will be increased. We can iteratively include new states and solve the relaxed dual problem until all states has $v^{*} \geq \sum_{i} w_{i}^{*} r_{i, k}$. The resulting $v^{*}$ will be the optimal solution for the original dual. The detailed algorithm is shown in Fig. 1.

Define the weighted-sum-rate for state $j$ as $\sum_{i} w_{i}^{*} r_{i, j}$. As removing non-active constraints in (9) will not change the optimal value of the relaxed dual, we can remove states with a weighted-sum-rate smaller than $v^{*}$ from the set $\mathcal{J}$ 


\section{Iterative Optimization Algorithm}

01: Initialize $\mathcal{J}=\{0\}$.

02: Find the optimal $\left[\begin{array}{ll}v^{*} & \mathbf{w}^{*}\end{array}\right]$ of the LP problem in (9) (11) with constraints correspond to $\mathcal{J}$.

03: Search for a state $k$ with $v^{*}<\sum_{i} w_{i}^{*} r_{i, k}$.

04: if $k$ exists

05: $\quad$ Set $\mathcal{J}=\{k\} \cup \mathcal{J}$.

06: $\quad$ Set $\mathcal{J}=\mathcal{J} \backslash\{j\}$, for all $j \in \mathcal{J}$ in which $v^{*}>\sum_{i} w_{i}^{*} r_{i, j}$.

07: $\quad$ Go to step 02 .

08: else

09: Optimize the LP in (6) - (8) while setting

10: endif $r_{i, j}=0, \forall j \notin \mathcal{J}$ and output the schedule.

Fig. 1. Iterative Optimization problem.

after each iteration. In this case, non-active states will be constantly removed and there will be $O(M)$ active states in each iteration, so the optimization of the relaxed dual can be carried out efficiently. As shown in [19], the cutting plane method is equivalent to the delayed column generation method on the prime problem. Therefore, the algorithm is guaranteed to converge and the procedure is essentially a revised Simplex pivoting process.

\section{Separation Problem}

The separation problem in the cutting plane method is the sub-problem of finding a state $k$ with a weighted-sum-rate larger than $v^{*}$ in step 03 of the iterative optimization algorithm.

One way to solve the separation problem is via exhaustively searching all the $2^{M}$ states and find the state $k$ with maximum value of weighted-sum-rate. Although exhaustive searching can always find the state with a weighted-sum-rate larger than $v^{*}$ when such state exists, the computation complexity of this method is clearly $\Omega\left(2^{M}\right)$.

We can use the knowledge on the relationship between reception rates and states in the optimization to reduce the complexity of the searching process. We observe that $w_{i}^{*}$ is the weight for the receiving rate at node $i$. If the solution of the relaxed dual problem contains weights $w_{l}^{*}$ which are equal to zero, then the receiving rate of node $l$ should not be the bottleneck for the relay chain. Suppose node $l$ listens in some state $k$. We can construct a new state $k^{\prime}$ in which $l$ is transmitting and all other nodes' listening/transmitting modes is the same as state $k$. Note that changing the state of node $l$ to transmitting will reduce the rate $r_{l, k^{\prime}}$ to zero. However, the rate of all the other nodes will not decrease, i.e., $r_{i, k} \leq r_{i, k^{\prime}}, \forall i \neq l$, as the transmitting node set $\mathcal{T}\left(k^{\prime}\right)=\mathcal{T}(k) \cup\{l\}$. Since we have $w_{l}^{*}=0$, we get $\sum_{i} w_{i}^{*} r_{i, k} \leq \sum_{i} w_{i}^{*} r_{i, k^{\prime}}$. In other words, state $k^{\prime}$ always has a higher weighted-sum-rate than state $k$. So, we only need to search states which sets all nodes $l$ as transmitting when the corresponding $w_{l}^{*}=0$.
We can also use local search heuristic algorithm to improve the search speed. A $d$-opt local search algorithm only searches states $k^{\prime}$ which have at most $d$ nodes change their states from the original state of $k$. In our problem, we know states which correspond to active constraints in the relaxed dual in (9) will have a weighted-sum-rate equal to $v^{*}$. Therefore, these states can serve as the starting states of the local search. If one of the neighboring states of these states has a weighted-sum-rate larger than $v^{*}$, we will move to this new state and search its neighborhood in order to find a state with even higher weighted-sum-rate. We will repeat the searching until no state in the neighborhood has a higher weighted-sum-rate or we have moved to more than $M$ states. The $d$-opt local search algorithm only needs to search $O\left(M^{d}\right)$ neighbors of $O(M)$ states, so the algorithm terminates in polynomial time.

\section{Algorithm Evaluation}

Although the local search algorithm can be trapped in some local optimal points, we show in our numerical experiments that the algorithm can still provide good approximations with small searching neighborhoods.

We generate randomly deployed networks to evaluate our algorithm. In the experiments, nodes are placed in a square network with side length of $M+1$. The source node 0 is placed at the middle point $\left(\frac{M+1}{2}, 0\right)$ of one side of the square and the destination node $M+1$ is placed at the middle point $\left(\frac{M+1}{2}, M+1\right)$ of the opposite side. $M$ relays are randomly deployed in the square to relay data from the source to the destination.

For each network size, we randomly generate 1000 network topologies and run the iterative algorithm to find the relay schedule. The number of iterations used by the 2-opt algorithm is shown in Fig. 2. We see that for a relay network with moderate size (less than 40 relays), we need about 500 iterations to solve the scheduling problem on average. Via linear fitting, we find that the number of iterations increases with $M$ as $O\left(M^{1.87}\right)$.

We also compare the performance of the $d$-opt local search heuristic algorithm with the optimal exhaustive search in random topologies. Fig. 3 shows the probability that the heuristic returns a suboptimal result when using different searching neighborhood size of $d$. We see that when using 1-opt local search, i.e., switching the transmission state of a single node in the searching process, the chance of returning a suboptimal result is quite high (up to nearly $80 \%$ ). When the neighborhood size increases to 2 , the chance of returning a local maximal point has been greatly reduced.

Define the approximation ratio $\rho$ as the ratio between the suboptimal solutions returned by the heuristic to the optimal solutions. Fig. 4 shows the approximation ratio of the heuristic, where the approximation ratio is averaged over topologies where the heuristic returns a suboptimal result. For the 1-opt local search, the approximation ratio is around 0.9997 for all network sizes. This shows that the suboptimal results returned by the heuristic are very close to the optimal one. Unlike the probability of returning a suboptimal point, which increases 


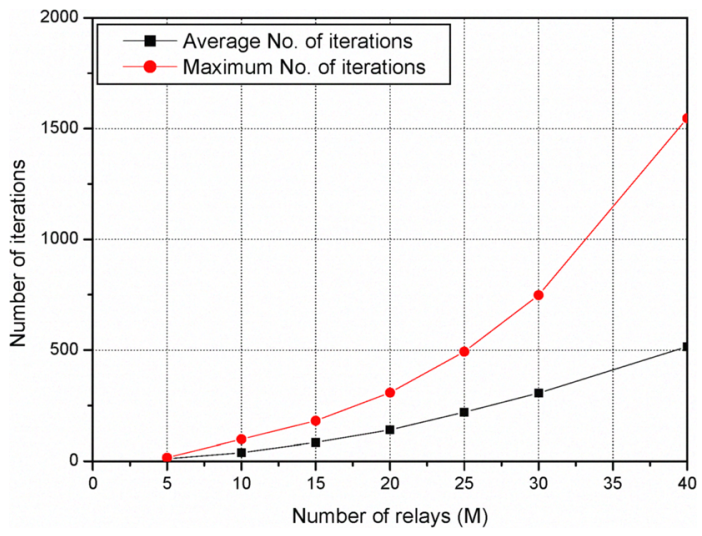

Fig. 2. Average and maximum number of iterations used by the iterative optimization algorithm (2-opt heuristic).



Fig. 3. Probability that the heuristic returns a suboptimal result.

with the number of relays, the approximation ratio remains flat as the network size increases. Thus, the 1-opt heuristic could be a good approximation even for large networks as the difference between the suboptimal result and optimal result is only about $0.03 \%$. When the searching neighborhood increases to 2-opt, we see that the difference between the suboptimal results and optimal results can be further reduced.

\section{E. Extending to Multiple Source-Destination Pairs}

In the previous discussions, we assume that there is only one source/destination pair in the network. In this section, we relax this assumption and extend the linear programming formulation to solve networks with multiple sources and destinations.

Consider the case that there are $K$ source-destination pairs, where source nodes are $0,1, \ldots, K-1$ and destination nodes are $K+M, K+M+1, \ldots, 2 K+M-1$. Suppose there are $M$ relays, which are nodes $K, K+1, \ldots, K+M-1$,

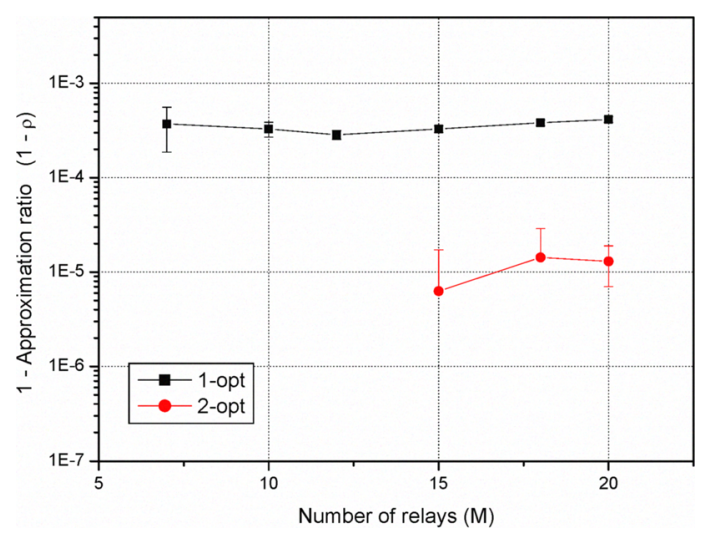

Fig. 4. The difference between suboptimal results and the optimal value (confidence interval 95\%).

in the area. The route for source-destination pair $k$ is $\mathcal{O}_{k}=$ $\left\{o_{k, 0}, o_{k, 1}, \ldots, o_{k,\left|\mathcal{O}_{k}\right|-1}\right\}, 0 \leq k \leq K-1$, where $o_{k, 0}=k$ is the source of the route and $o_{k,\left|\mathcal{O}_{k}\right|-1}=K+M+k$ is the destination of the route. In this section, we assume that each relay only appears once in any route and source/destination nodes cannot act as relays.

In the multiple source/destination pairs case, concurrent transmissions in different routes can interfere with each other. So, the source nodes should also toggle between transmitting and listening states to avoid interfering with nodes in other routes. However, we can still let all destinations to be always listening. Therefore, the transmission state vector can be defined as $\mathbf{s}=\left(s_{0} s_{1} \ldots s_{K+M-1}\right)$ and the number of transmission state is increased to $2^{K+M}$ in this case.

The receiving rate of node $o_{k, i}$ at state $j$ can be written as:

$$
r_{o_{k, i}, j}=\left\{\begin{array}{r}
\log \left(1+\frac{\sum_{l \in\left(o_{k, 0}, \ldots, o_{k, i-1}\right) \cap \mathcal{T}(j)} \lambda_{o_{k, i}, l} P_{l}}{N_{o_{k, i}}+\sum_{l \in \mathcal{T}(j) \backslash \mathcal{O}_{k}} \lambda_{o_{k, i}, l} P_{l}}\right) \\
o_{k, i} \in \mathcal{L}(j) \\
0 \quad o_{k, i} \in \mathcal{T}(j)
\end{array}\right.
$$

To achieve max-min fairness over all source destination pairs, the optimal rate can be calculated through the following linear programming problem:

$$
\begin{gathered}
\text { s.t. } u-\sum_{j=0}^{2^{K+M}-1} q_{j} r_{i, j} \leq 0 \quad K \leq i \leq 2 K+M-1 \\
\sum_{j=0}^{2^{K+M}-1} q_{j}=1 \\
q_{j} \geq 0 .
\end{gathered}
$$

Constraint (14) requires all relays and destinations should receive at a rate not smaller than $u$. By maximizing $u$, we are trying to maximize the minimal rate achieved by all source/destionation pairs. 
Similar to the single source/destination case, this problem can be solved via the cutting plane method. Note that when there are no relays, i.e., $M=0$, the problem will be reduced to throughput optimization problem in wireless networks, which is NP-complete in general [3].

\section{NumeriCAL RESUlts}

\section{A. Performance Comparison}

In this section, we compare the performance of the halfduplex decode-forward strategy with the cut-set upper bound in information theory [20] and the multi-hop ( $\mathrm{MH}$ ) relay strategy used in conventional wireless networks. The comparison is based on the numerical method developed in previous sections.

\section{Cut-set bound:}

The cut-set upper bound provides an upper bound on the transmission rate for all possible transmission schemes in a multi-terminal network [20]. The cut-set bound in the MRC can be derived as follows. We arbitrarily partition the node set $\mathcal{M}$ into two subsets, $\mathcal{B}$ and its complement $\mathcal{B}^{c}$. Suppose node $i$ can send information at $R_{i, j}$ to node $j$. Then, the total information flow across the cut should be bounded by [20]:

$$
\sum_{i \in \mathcal{B}, j \in \mathcal{B}^{c}} R_{i, j} \leq I\left(X_{\mathcal{B}} ; Y_{\mathcal{B}^{c}} \mid X_{\mathcal{B}^{c}}\right)
$$

for all $\mathcal{B} \subset \mathcal{M}$, where $I\left(X_{\mathcal{B}} ; Y_{\mathcal{B}^{c}} \mid X_{\mathcal{B}^{c}}\right)$ is the mutual information between the signal sent by nodes in $\mathcal{B}$ (denoted as $X_{\mathcal{B}}$ ) and signal received by nodes in $\mathcal{B}^{c}$ (denoted as $Y_{\mathcal{B}^{c}}$ ) when the signal sent by nodes in $\mathcal{B}^{c}$ (denoted as $X_{\mathcal{B}^{c}}$ ) is known.

For the phase fading Gaussian MRC, it can be shown that the upper bound of the information rate that the source can send to the destination is [18]:

$$
R_{\mathrm{UB}}=\max _{\mathcal{Q}} \min _{\substack{\mathcal{B} \subset \mathcal{M} \\\{0\} \subseteq \mathcal{B} \&\{M+1\} \subseteq \mathcal{B}^{c}}} R_{\mathcal{B}},
$$

where

$$
R_{\mathcal{B}}=\sum_{j=0}^{2^{M}-1} q_{j} \log \left(1+\sum_{i \in \mathcal{B}^{c} \cap \mathcal{L}(j)} \frac{\sum_{k \in \mathcal{B} \cap \mathcal{T}(j)} \lambda_{i, k} P_{k}}{N_{i}}\right)
$$

Similar to the max-min problem in Sec. III, the max-min problem in (18) can be formulated as a linear programming problem. However, the possible number of cuts with $\{0\} \subseteq \mathcal{B}$ and $\{M+1\} \subseteq \mathcal{B}^{c}$ is $2^{M}$. Therefore, the linear programming has both $O\left(2^{M}\right)$ constraints and variables. The size of problems that is solvable in this case is thus quite limited.

\section{Multi-hop relay:}

In conventional wireless ad hoc networks, relay nodes do not cooperatively send the information. Packets are sent in a hop-by-hop manner where each hop is treated as a point-topoint link [21]. In this strategy, commonly known as the multihop relay strategy (MH), node $i$ only listens to its immediate neighbor in the upstream, node $i-1$, and all signals from other relays will be treated as noises.

The maximum achievable rate for this strategy can be derived in a way similar to the single source/destination

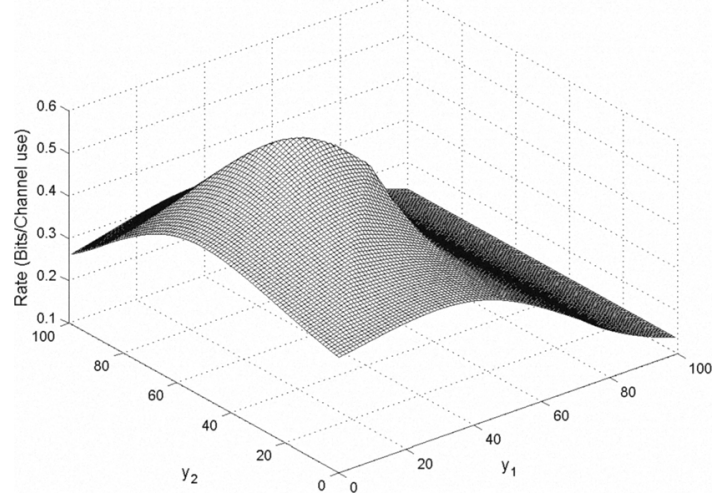

Fig. 5. DF rates for the two-relay MRC with varying relay positions.

pair problem in Sec. III. Suppose the source is node 0, destination is node $M+1$ and node $1,2, \ldots, M$ are relays. The transmission state vector will be defined as $\mathbf{s}=\left(s_{0} s_{1} \ldots s_{M}\right)$ and the receiving rate of node $i$ at state $j$ can be written as:

$$
r_{i, j}=\left\{\begin{array}{c}
\log \left(1+\frac{\lambda_{i-1, i} P_{i-1}}{N_{i}+\sum_{k \in \mathcal{T}(j) \backslash\{i-1\}} \lambda_{k, i} P_{k}}\right) \\
i \in \mathcal{L}(j) \& \quad i-1 \in \mathcal{T}(j) . \\
0 \quad \text { otherwise }
\end{array}\right.
$$

In this case, any concurrent transmissions will interfere with each other. The problem of maximizing the $\mathrm{MH}$ rate is also a max-min problem and can be solved via linear programming.

\section{B. Networks with Two Relays}

We first consider a simple case, where the network has two relays which are placed on a straight line connecting the source and destination. The node coordinates are: node $0(0,0)$, node $1\left(0, y_{1}\right)$, node $2\left(0, y_{2}\right)$, and node $3(0,100)$. The position of relay nodes can be changed via selecting $y_{1}, y_{2} \in[0,100]$. As stated earlier, we assume that the route is fixed as $\{0,1,2,3\}$. We find an optimal schedule that maximizes the DF rate for this route, although it might not be the optimal route for all topologies. We assume that $\kappa=1, \eta=2, P_{i}=10$ for $i=$ $0,1,2$, and $N_{j}=0.01$, for $j=1,2,3$.

Fig. 5 shows the DF rates for the two-relay half duplex phase fading Gaussian MRC with varying relay positions. Fig. 6 shows the capacity upper bound and the difference between the upper bound and the achievable rates of DF.

The highest DF rate is 0.5956 bits/channel use, when $y_{1}=$ $34, y_{2}=56$. The optimal schedule for this topology is $q_{0}=$ $0.0038, q_{1}=0.6585, q_{2}=0.3377, q_{3}=0$. The worst $\mathrm{DF}$ rate is $0.1375 \mathrm{bits} / \mathrm{channel}$ use, when $y_{1}=100$ (relay 1 at the destination). The optimal schedule for this topology is $q_{0}=$ $1, q_{1}=q_{2}=q_{3}=0$, i.e., nodes 1 and 2 only listen. For the capacity upper bound, the highest rate is $0.7080 \mathrm{bits} / \mathrm{channel}$ use, when $y_{1}=35, y_{2}=65$ or $y_{1}=65, y_{2}=35$ (note the symmetry). The lowest rate is 0.3757 bits/channel use, when $y_{1}=0, y_{2}=0$ or $y_{1}=100, y_{2}=100$, i.e., when both relays are at the source or at the destination. 


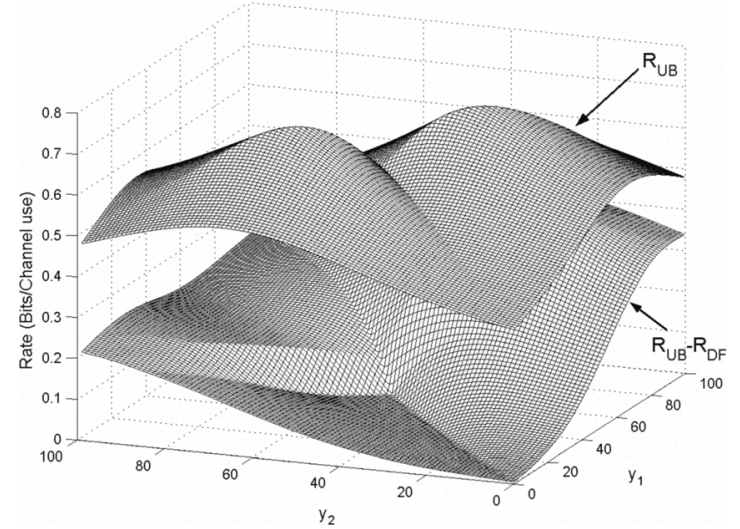

Fig. 6. Capacity upper bound and the gap between cut-set upper bound and achievable rates.

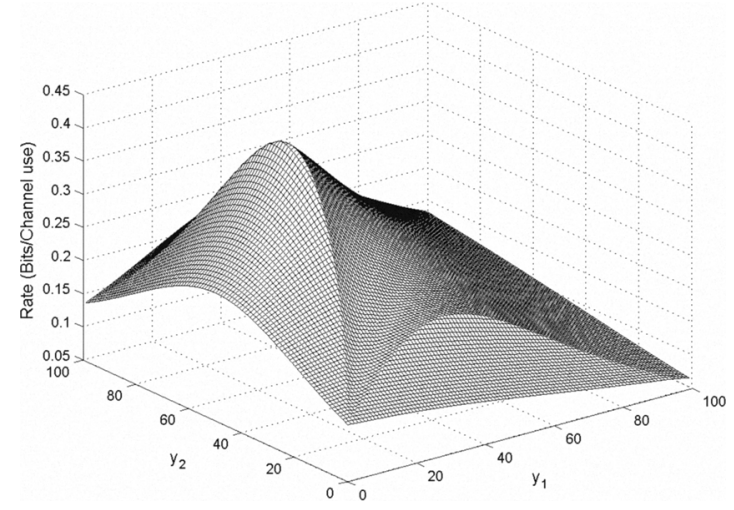

Fig. 7. Multi-hop rates for the two-relay MRC with varying relay positions.

Unlike the full duplex case where DF achieves the cut-set upper bound and hence achieves the capacity when the relays are within certain distances from the source [7], DF in the half duplex case does not achieve (though close to) the cut-set bound if a relay is $\epsilon$ away from the source, for any arbitrarily small distance $\epsilon>0$.

Fig. 7 shows the rates for $\mathrm{MH}$ strategy $\left(R_{\mathrm{MH}}\right)$. The highest MH rate is 0.4105 bits/channel use, when $y_{1}=29, y_{2}=62$ and the worst $\mathrm{MH}$ rate is $0.0671 \mathrm{bits} / \mathrm{channel}$ use, when $y_{1}=$ 100 and $y_{2}=0$. From Fig. 8, which shows the difference between the DF and MH rates, we observe that the achievable DF rate is always higher than the MH rate. When both relays are at the position of the destination, i.e., $y_{1}=y_{2}=100$, DF and $\mathrm{MH}$ have nearly the same performance. Averaged over the possible positions of relay 1 and 2, the DF rate is $76.35 \%$ higher than the $\mathrm{MH}$ rate.

Fig. 9 shows optimal schedules when varying node 2's position for the DF scheme, i.e., value of $y_{2}$, while fixing the rest of the nodes' positions (node 1 is fixed at $(0,66)$ ). State 00 means both relay are listening, state 01 means node 1 is listening and node 2 is transmitting and state 10 means

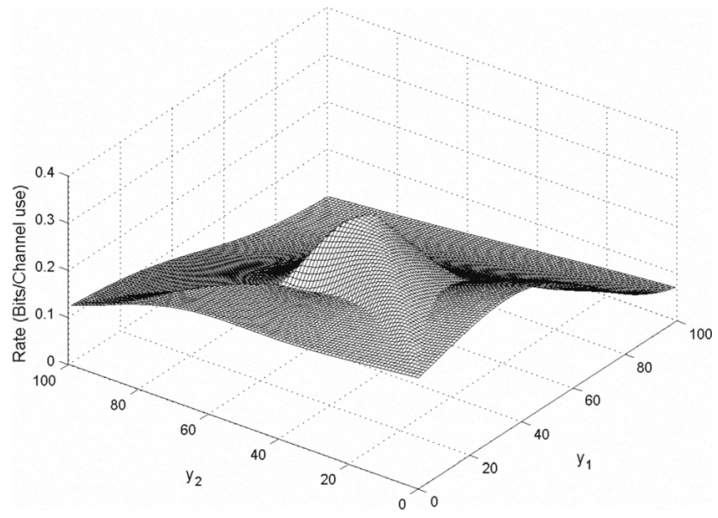

Fig. 8. $\quad R_{\mathrm{DF}}-R_{\mathrm{MH}}$, the gap between $R_{\mathrm{DF}}$ and $R_{\mathrm{MH}}$.

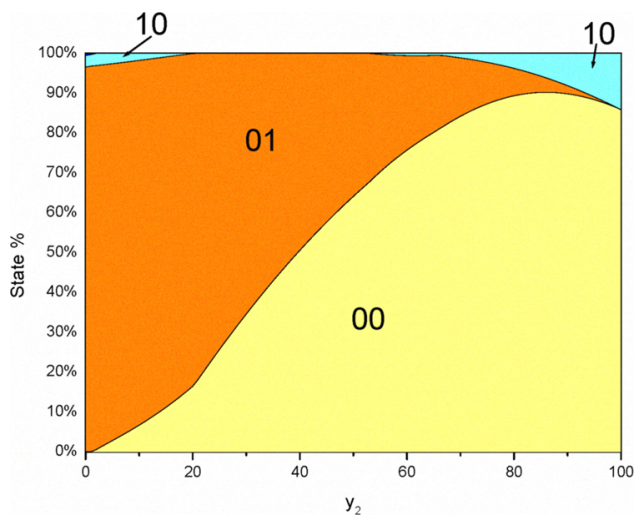

Fig. 9. Graph showing optimal schedules for $y_{1}=66$ and varying node 2's position in DF scheme.

node 1 is transmitting and node 2 is listening. We see that as node 2 moving towards the destination (increasing $y_{2}$ ), node 2 tends to spend more time in listening. We also see that at most 3 states are used in the optimal schedule. This verifies that the optimal schedule use at most $M+1$ states.

\section{Networks with a Chain Topology}

In this section, we consider networks with $M$ relays on a straight line. The coordinates of node $i$ are $(0, i)$ for $i=$ $0, \ldots, M+1$. Therefore, the distance between the source and destination will increase as the number of relays increases. We assume that $\kappa=1, \eta=2, P_{i}=P=10$ for $i=0, \ldots, M$, and $N_{j}=N=1$, for $j=1, \ldots, M+1$.

Fig. 10 shows the rate for a relay chain with different number of relays. As the source and destination become farther apart, the rate for DF strategy slightly reduces. However, the rate will finally converge to some constant when the distance between source and destination goes to infinity.

The lower bound for the achievable DF rate can be derived as follows. Assume that we use a schedule with two states. In the first state, only nodes $2 k+1, k=0, \ldots,\lfloor(M+1) / 2\rfloor$ are sending and other nodes are listening. In the second state, only 


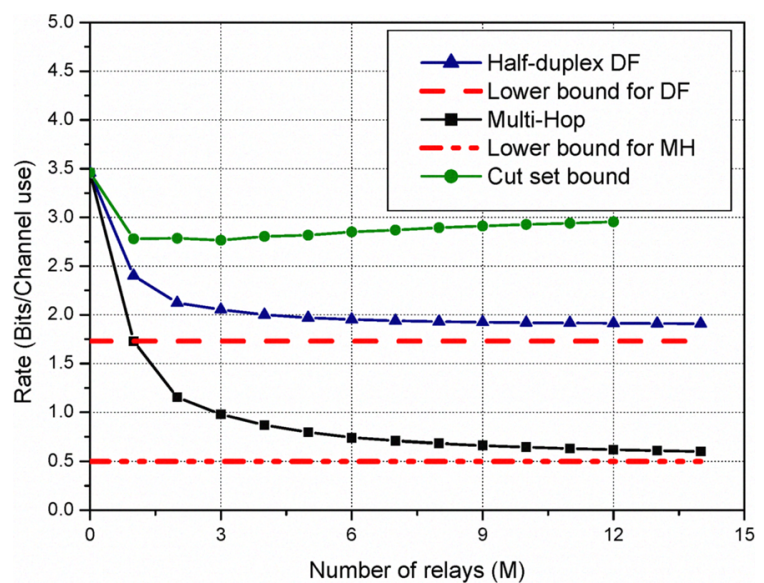

Fig. 10. Rates in chain topology for half-duplex DF and MH strategy.

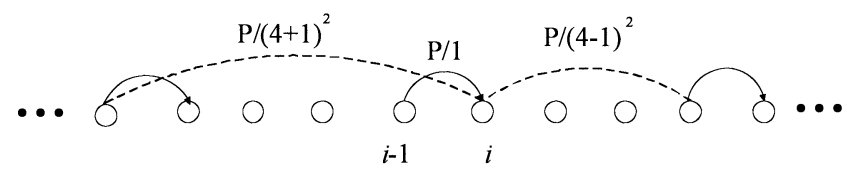

Fig. 11. Interference of the relays in a chain topology.

nodes $2 k$ are sending and nodes $2 k+1$ are listening. If relays spend equal times in these two states, we have the rate lower bound for the half-duplex strategy as $\log (1+P / N) \times \frac{1}{2}=$ 1.7297 bits/channel use, as shown in Fig. 10.

Fig. 10 also shows the cut-set rate upper bound. The upper bound first drops when we add the first relay due to the half duplex constraints. After that, the upper bound increases as the number of relays increases. For an infinitely long chain of relays, we can see that the rate is bounded by the cut between the source and the reset of nodes, i.e., setting $\mathcal{B}=\{0\}$ and $\mathcal{B}^{c}=\{1,2, \ldots, M+1\}$. For this cut, the upper bound for rate $R_{\mathcal{B}}$ can be calculated from (19) when only node 0 is sending:

$$
R_{\mathcal{B}} \leq \log \left(1+\sum_{i=1}^{M+1} \frac{P / i^{2}}{N}\right)
$$

when we assume $\eta=2$. Using the fact that $\sum_{i=1}^{\infty} \frac{1}{i^{2}}=\frac{\pi^{2}}{6}$, we see that the cut set upper bound should be smaller than $\log \left(1+\frac{\pi^{2}}{6} \frac{P}{N}\right)=4.1251$ bits/channel use when $M \rightarrow \infty$. This upper bound is about 2.38 times of the achievable DF rate. However, this cut-set upper bound could be quite loose and not achievable for any relay strategy.

The end-to-end rate of $\mathrm{MH}$ also drops as the distance between the source and destination increases. The lower bound for MH strategy can be derived as follows. We can use a four-state schedule. In the first state, nodes $4 k+1, k=$ $0, \ldots,\lfloor(M+1) / 4\rfloor$ are sending and all other nodes are listening. Nodes $4 k+2,4 k+3$ and $4 k$ sends at the second, third and fourth state consequently. When we have a long chain of nodes with $M \rightarrow \infty$, the receiving node in each state will have rate of:

$$
\log \left[1+\frac{P / 1^{2}}{N+\sum_{k=1}^{\infty} \frac{P}{(4 k-1)^{2}}+\sum_{k=1}^{\infty} \frac{P}{(4 k+1)^{2}}}\right],
$$

see Fig. 11.

We have

$$
\begin{aligned}
& \sum_{k=1}^{\infty} \frac{P}{(4 k-1)^{2}}+\sum_{k=1}^{\infty} \frac{P}{(4 k+1)^{2}} \\
= & P\left(\sum_{k=1}^{\infty} \frac{1}{k^{2}}-1-\sum_{k=1}^{\infty} \frac{1}{(2 k)^{2}}\right) \\
= & P\left(\frac{3}{4} \sum_{k=1}^{\infty} \frac{1}{k^{2}}-1\right) \\
= & P\left(\frac{\pi^{2}}{8}-1\right) .
\end{aligned}
$$

Therefore, the achievable end-to-end rate will be:

$$
\frac{1}{4} \log \left[1+\frac{P}{N+P\left(\frac{\pi^{2}}{8}-1\right)}\right]
$$

when we spend equal time in the four states. This rate is shown on Fig. 10 as the lower bound of the MH scheme. It can be verified that this rate is larger than the rate of using three states which alternately ask every third node to send.

As the $\mathrm{MH}$ rate always decreases when we increase the distance between source and destination, the optimal rate for $\mathrm{MH}$ under a chain length of $M=12$, which is 0.6104 bits/channel use, can serve as an upper bound for the achievable rate for a chain with $M \geq 12$. Therefore, we can see that the lower bound for half-duplex DF scheme on an infinity long chain is nearly 2.8 times larger than the upper bound of the $\mathrm{MH}$ scheme. This shows the benefit of using cooperative relay schemes in a multi-hop network.

\section{Randomly Deployed Networks}

The structure of randomly deployed networks used in this section is same as in Sec. III-D. Relays are randomly deployed in a square area of side length $M+1$ while the source and the destination are fixed at the middle point of opposite sides. We assume that $\kappa=1, \eta=2, P_{i}=10$ and $N_{j}=1$ in this section.

Fig. 12 shows the rate for $\mathrm{DF}$ and $\mathrm{MH}$, averaged over 1000 topologies. Similar to the chain topology, both DF and $\mathrm{MH}$ rates reduce as the network size increases. However, we see that the rate for randomly deployed networks is much smaller than the chain topology. This can be explained via Figs. 5 and 7 , which show that placing relays with equal space on the straight line connecting the source and destination is close to optimal for both DF and $\mathrm{MH}$.

Fig. 12 shows the gap between DF rate and MH rate is increasing when the number of relays increases. In random networks, the MH rate is limited by the longest hop, which increases in larger networks due to the randomness in deployment. For DF strategy, multiple nodes can help each other to 


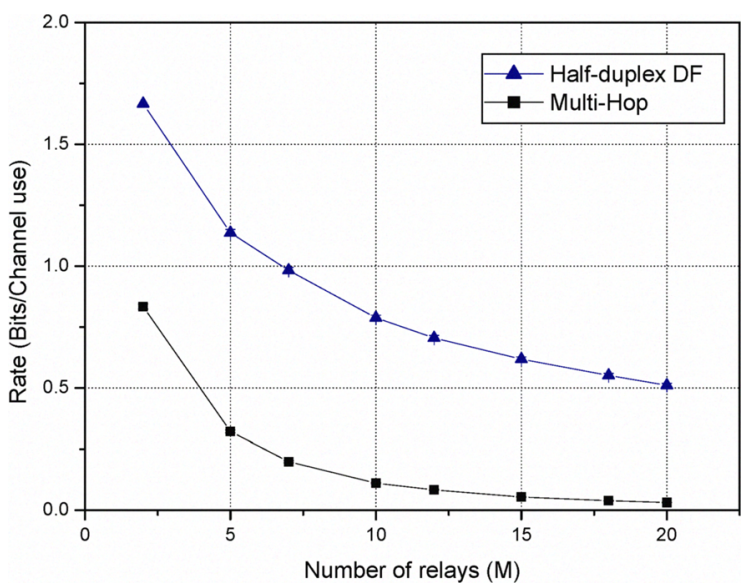

Fig. 12. Rates in randomly deployed network for half-duplex DF and MH strategy.

send message across the gap. So, the MH rate decreases to nearly zero when the network size increases to 20 while DF strategy can still maintain a decent rate.

\section{CONCLUSION}

We studied the transmission schedule optimization problem for half-duplex multiple relay channels. The schedule optimization problem can be formulated as a linear programming problem for the phase fading Gaussian MRC. We showed that only a small amount of transmission states need to be considered in the optimal schedule. We also proposed a heuristic which can provide good approximations for the schedule optimization problem. The results presented in this paper showed that cooperative coding scheme can be a promising way to increase the throughput of multi-hop wireless networks.

\section{REFERENCES}

[1] P. Gupta and P. R. Kumar, "The capacity of wireless networks," IEEE Trans. Inf. Theory, vol. 46, no. 2, pp. 388-404, Mar. 2000.

[2] M. Grossglauser and D. N. C. Tse, "Mobility increases the capacity of ad hoc wireless networks," IEEE/ACM Trans. Networking, vol. 10, no. 4, pp. 477-486, Aug. 2002.

[3] K. Jain, J. Padhye, V. N. Padmanabhan, and L. Qiu, "Impact of interference on multi-hop wireless network performance," in Proc. ACM Mobicom, Sep 2003.

[4] T. M. Cover and A. A. El Gamal, "Capacity theorems for the relay channel," IEEE Trans. Inf. Theory, vol. IT-25, no. 5, pp. 572-584, Sep. 1979.

[5] A. Ozgur, O. Leveque, and D. Tse, "Hierarchical cooperation achieves optimal capacity scaling in ad hoc networks," IEEE Trans. Inf. Theory, vol. 53, no. 10, pp. 3549-3572, Oct. 2007.

[6] L. Xie and P. R. Kumar, "An achievable rate for the multiple level relay channel," IEEE Trans. Inf. Theory, vol. 51, no. 4, pp. 1348-1358, Apr. 2005.

[7] G. Kramer, M. Gastpar, and P. Gupta, "Cooperative strategies and capacity theorems for relay networks," IEEE Trans. Inf. Theory, vol. 51, no. 9, pp. 3037-3063, Sep. 2005.

[8] L. Ong and M. Motani, "Myopic coding in multiterminal networks," IEEE Trans. Inf. Theory, vol. 54, no. 7, pp. 3295-3314, Jul. 2008.

[9] A. E. Gamal and S. Zahedi, "Capacity of a class of relay channels with orthogonal components," IEEE Trans. Inf. Theory, vol. 51, no. 5, pp. 1815-1817, May 2005.
[10] A. Host-Madsen, "On the capacity of wireless relaying," in Proc. of IEEE Semiannual Vehicular Technology Conference (VTC 2002), Birmingham, Al, May 6-9 2002.

[11] M. A. Khojastepour, A. Sabharwal, and B. Aazhang, "On the capacity of 'cheap' relay networks," in Proc. Conf. on Info. Science and Sys. (CISS), Baltimore, MD, Mar. 12-14 2003.

[12] G. Kramer, "Models and theory for relay channels with receive constraints," in Proc. 42nd Allerton Conf. on Commun., Control, and Comput., Monticello, IL, Sep. 29-Oct. 12004.

[13] A. Host-Madsen and J. Zhang, "Capacity bounds and power allocation for the wireless relay channel," IEEE Trans. Inf. Theory, vol. 51, no. 6, pp. 2020-2040, Jun. 2005.

[14] P. Gupta and P. R. Kumar, "Towards an information theory of large network: an achievable rate region," IEEE Trans. Inf. Theory, vol. 49, no. 8, pp. 1877-1894, Aug. 2003.

[15] E. C. van der Meulen, "Three-terminal communication channels," $A d v$. Appl. Prob., vol. 3, pp. 120-154, 1971.

[16] L. Ong and M. Motani, "Optimal routing for the decode-and-forward strategy in the Gaussian multiple relay channel," in Proc. IEEE Int. Symposium on Inf. Theory (ISIT), Nice, France, Jun. 24-29 2007, pp. 1061-1065.

[17] — "Optimal routing for decode-and-forward based cooperation in wireless network," in Proc. 4th Annu. IEEE Conf. on Sensor and Ad Hoc Commun. and Netw. (SECON), San Diego, CA, Jun. 18-21 2007, pp. 334-343.

[18] L. Ong, W. Wang, and M. Motani, "Achievable rates and optimal schedules for half duplex multiple-relay networks," in Proc. 46th Allerton Conf. on Commun., Control, and Comput., Sep 2008.

[19] D. Bertsimas and J. N. Tsitsiklis, Introduction to Linear Optimization. Athena Secintific., 1997.

[20] T. M. Cover and J. A. Thomas, Elements of Information Theory. John Wiley and Sons, 1991.

[21] J. Li, C. Blake, D. S. J. D. Couto, H. I. Lee, and R. Morris, "Capacity of ad hoc wireless networks," in Proc. ACM Mobicom, Rome, Italy, Jul. 16-21 2001. 